\title{
Contact Measurement of Thermal Conductivity and Thermal Diffusivity of Solid Materials: Experimental Validation of Feasibility with a Prototype Sensor
}

\author{
Syamsul Hadia, Mamoru Nishitani ${ }^{a}$, Agung Tri Wijayanta ${ }^{\text {b }}$, Takanobu Fukunaga ${ }^{a}$, \\ Kosaku Kurata ${ }^{a}$ and Hiroshi Takamatsu ${ }^{a}$ \\ a Department of Mechanical Engineering, Kyushu University \\ 744 Motooka, Nishi-ku, Fukuoka, 819-0395, Japan \\ E-mail: takamatsu@mech.kyushu-u.ac.jp \\ b Research and Education Center of Carbon Resources, Kyushu University \\ 6-1 Kasuga-koen, Kasuga, Fukuoka 816-8580, Japan
}

\section{Highlights:}

$>$ A method for measuring thermal transport properties using a novel stamp sensor.

$>$ It can use for in-situ non-invasive contact measurement

$>$ It has an advantage in measuring soft materials.

$>$ Thermal contact resistance is eliminated using certain thickness gel layer.

$>$ Thermal conductivity indicates to be determined within $10 \%$ errors.

\footnotetext{
* Corresponding author: Tel./Fax.: +81 928023123

E-mail address: takamatsu@mech.kyushu-u.ac.jp (Hiroshi Takamatsu)
} 


\begin{abstract}
A contact method has been proposed for measuring thermal transport properties of solids including soft materials. The method has an advantage of potential utilization for in-situ measurement without preparing a sample specimen. A unique feature of the method is to prepare a shallow cavity around a film sensor for a layer of a gel that is used to eliminate the thermal contact resistance between the sensor and the sample. A prototype sensor, $3 \mathrm{~mm}$ in diameter, was fabricated on the surface of $0.16-\mathrm{mm}$ thick glass substrate, and used with a $50-\mu \mathrm{m}$ thick silicon rubber sheet as a spacer for the gel. The transient temperature rise of the sensor was determined from the electrical resistance after heating the sensor at a constant current. The thermal conductivity and the thermal diffusivity of a sample as well as the thickness of the gel layer were then determined from an iteratively obtained theoretical temperature rise that agreed with the measured temperature rise. The results obtained the experiments with four different materials indicated that the thermal conductivity could be determined within $10 \%$ errors. The present study therefore demonstrates feasibility of the method, while improvement is still needed to reduce the error particularly in the thermal diffusivity.
\end{abstract}

Keywords: measurement technique, thermal conductivity, thermal diffusivity, contact measurement, in-situ measurement 


\section{INTRODUCTION}

Thermal transport properties of solid materials are measured with some commercially available devices with different principles and methods. Application of these methods depends on the sample material and conditions. When we measure the thermal diffusivity as standard reference data particularly at extreme conditions, we presumably use the laser flash method. In this method, the temperature change at a surface of a specimen is measured after pulse-heating at its back side by laser irradiation [1-4]. It has an advantage in terms of the accuracy, but it requires preparation of a specimen with precise thickness. It also requires coating of the specimen to absorb the laser power at the surface when the sample is transparent or semi-transparent to the laser light. When we need the data for a poorly conducting material, we may use the transient plane source method, which measures the temperature of the heater, a hot-disk or a hot-strip, that are sandwiched between two pieces of samples [5-12]. It has been used for a variety of materials [13-19], but clamping the sensor strongly with the samples after polishing surfaces $[13,14]$, sometime with a small amount of liquid in between [6], is crucial for reducing the thermal contact resistance between the sample and the sensor. It also requires two identical specimens. For non-invasive, in-situ measurement of solid materials, we can apply the point contact-probe method. The thermal transport properties are obtained with only pressing a small spherical heated probe onto the surface of a sample material [20-22]. However, the effect of thermal contact resistance between the material and the probe on the measurement is also inevitable in this case as well. To reduce its effect, the probe is pressed at a constant pressure.

Although these methods, which have advantages and limitations, are to be used for a variety of materials, none of these is appropriate for soft materials including biological materials. Preparing a specimen of soft material with precise dimension is difficult in the laser flash method. Clamping soft specimens hard without deformation is difficult in the 
transient plane method. Pressing a bead probe on a soft sample would alter the contact area in the point contact-probe method, although the method assumes point contact between the sensor and the sample.

Hence to develop a convenient method for measuring solids including soft materials, we have proposed a new contact method using a 'stamp-type sensor' [23]. The thermal transport properties are measured by pressing a small thin film sensor that was fabricated on a surface of a thin plate. It could therefore be used for non-destructive, in-situ measurement. The uniqueness of this method is to put a gel between the sensor and a sample to eliminate the thermal contact resistance. In addition, a shallow cavity with given dimensions has been prepared around the sensor for the gel. The feasibility of the method to determine the thermal conductivity and the thermal diffusivity has been checked using virtual experimental data that have been generated by adding an artificial scattering to a theoretical temperature change of the sensor [23]. The results indicated that the thermal conductivity was determined within the error less than $\sim 2 \%$ and the thermal diffusivity within $\sim 5 \%$ error. Since the theoretical examination showed that the method did work, the next step is to demonstrate the measurement by experiments. In this paper, we present preliminary results obtained by using a prototype sensor with several samples, i.e. acrylic resin, agar gel, machinable ceramic, and stainless steel.

\section{SENSOR AND METHODS}

\subsection{Sensor}

A conceptual design of the prototype stamp sensor is shown in Fig. 1. A thin metallic film heater that works as a resistance thermometer as well is deposited on the top of a glass substrate. A circular sensor is used so that the system could be described by an axisymmetric 2-D model. The sensor is pressed against the surface of a sample with a gel spread on the 
sensor to eliminate the contact thermal resistance between the sensor and the sample. The important feature of the sensor is that the sensor is placed at the bottom of a shallow cavity made by a spacer between the substrate and the sample, which roughly determines the thickness of the gel layer irrespective of the contact pressure.

The final design of the sensor would be a stamp-type device where a sensor is fabricated on the bottom of a cylindrical holder (Fig. 1). However, as a preliminary study, a platinum film of a pattern of the sensor and electrodes, $53 \mathrm{~nm}$ in thickness, was fabricated on a 0.16-mm thick $22 \times 40 \mathrm{~mm}^{2}$ glass substrate by physical vapor deposition (PVD) (Fig. 2). A circular pattern of $3 \mathrm{~mm}$ in diameter was drawn with a single stroke of a $125 \mu \mathrm{m}$ wide line. A $50-\mu \mathrm{m}$ thick annular silicone rubber sheet with a $15-\mathrm{mm}$ diameter hole was attached on the opposite side of the glass substrate as a spacer to provide a shallow cavity for the gel. The glass substrate was held with a hollow cylinder (Fig. 3). A sample was heated through the substrate, not directly by the sensor, to protect the sensor during cleaning up of the gel after experiments, even though the sensor was coated by a glass layer.

\subsection{Measurement}

Four different samples were prepared for measurement: an acrylic resin block of $40 \times 30 \times 15 \mathrm{~mm}^{3}$, a stainless steel SUS304 block of $40 \times 40 \times 30 \mathrm{~mm}^{3}$, and a machinable ceramic cylinder of $\varnothing 30 \times 20 \mathrm{~mm}^{3}$, and $1 \%$ agar gelated in a $20 \mathrm{~mm}$ deep plastic dish $100 \mathrm{~mm}$ in diameter. In the present experiment, the measuring device was set upside down to make it easy to handle taking into account its size and connected cables; a sample was put on the sensor-deposited glass plate that was held on the top of a hollow cylinder (Fig. 3).

The electrical resistance of the sensor was measured by a four-terminal method; the voltage drops in the sensor and a standard resistor connected to the power line were measured at every $0.18 \mathrm{~s}$ (Fig. 4). Prior to the experiments, the electrical resistance of the sensor was 
calibrated as a function of temperature by applying small currents to the sensor with keeping the sensor in a temperature-controlled dielectric liquid, FC-84.

A commercially available gel that is utilized in the medical ultrasonic echo was used in the experiment as an eliminator of thermal contact resistance. The sensor was pressed against a sample, i.e. the sample was put on the device actually, after spreading a small amount of gel in a shallow cavity. Measurement was started after waiting for the system to reach thermal equilibrium. A constant current at $0.1 \mathrm{~mA}$, which was negligibly small in regard of temperature rise of the sensor, was applied first for $5 \mathrm{~s}$ to measure the initial temperature of the system. The current was then increased stepwise to a prefixed value to heat the sensor. The measured voltage was recorded by a data acquisition system that was controlled by a computer. The heating rate was determined from our previous study that examined the measurement accuracy using numerical simulations [23]. The sensor was heated up to $\sim 5 \mathrm{~K}$ in the temperature rise within $5 \mathrm{~s}$ for all the materials.

\subsection{Theoretical analysis}

The physical model for the theoretical analysis is shown in Fig. 4. The system was defined by the cylindrical coordinate system with the origin at the center of the heater on the substrate surface.

The general description of heat conduction equation is

$$
\frac{\partial T}{\partial t}=\frac{1}{\rho c}\left[\frac{1}{r} \frac{\partial}{\partial r}\left(\lambda r \frac{\partial T}{\partial r}\right)+\frac{\partial}{\partial z}\left(\lambda \frac{\partial T}{\partial z}\right)\right]+\dot{q}_{v}
$$

where $\rho$ is the density, $c$ is the heat capacity and $\lambda$ is the thermal conductivity of each region.

The heat generation rate per unit volume $\dot{q}_{v}$ was incorporated only in the equation for the heater, and was assumed to be uniform and constant. The initial condition is

$$
T=T_{0} \quad \text { at } \quad t=0
$$


and the boundary conditions are described as follows:

$$
\begin{array}{ll}
T=T_{0} & \text { at } r \rightarrow \infty \text { or } z \rightarrow \infty \\
\partial T / \partial r=0 & \text { at } \quad r=0 \\
\partial T / \partial z=0 & \text { at } \quad z=-z 0
\end{array}
$$

The sensor side of the glass substrate, $z=-z 0$, that was exposed to the air inside the hollow cylinder was assumed to be adiabatic. The heat balance at the interfaces between different regions was also taken into account. The temperature change depends on the thermal conductivity through the continuity of heat flux at the interfaces, while Eq. (1) includes only $\alpha(=\lambda / \rho c)$ when the thermophysical properties were assumed to be constant independent of temperature.

Numerical solution to Eq. (1) was obtained by using a finite volume method with central difference for conduction terms and fully implicit formulation for unsteady terms. The solution domain was divided into $330 \times 1000$ meshes with variable sizes after examination of the effect of mesh size on the calculated result. Physical properties of water were used for the gel layer.

\subsection{Protocol to Determine Thermal Transport Properties}

Measurement of thermal transport properties includes a process to find the theoretical temperature rise that fits the experimental data, i.e. a process to determine the thermal conductivity $\lambda$, the thermal diffusivity $\alpha$ and the thickness $\delta$ of the gel layer which minimize the cumulative squared difference $S$ in the average temperature of the sensor between the theory and experiment:

$$
S=\sum_{i=1}^{N}\left(T_{e x p, i}-T_{c a l, i}(\lambda, \alpha, \delta)\right)^{2}
$$

where $N$ is the total number of measured data, $T_{\text {exp }, i}$ is the $i$-th measured temperature, and 
$T_{c a l, i}$ is the temperature obtained from the numerical solution corresponding to $T_{\text {exp,i }}$. The Gauss-Newton algorithm with a numerical approximation to the Jacobian was used for the nonlinear least-squares problems. The method used in a short-hot-wire method [24] to determine $\lambda$ and $\alpha$ was extended to a three-parameters problem. The algorithm is described as follows:

(1) Guess $\lambda, \alpha$ and $\delta$.

(2) Solve Eq. (1) numerically to obtain transient average temperature $T_{c a l, i}(\lambda, \alpha, \delta)$ $(i=1 \sim \mathrm{N})$.

(3) Solve Eq. (1) numerically to obtain three sets of temperatures for different combinations of $\lambda, \alpha$ and $\delta$, i.e. $T_{c a l, i}^{\prime}(\lambda+\Delta \lambda, \alpha, \delta), T_{c a l, i}^{\prime \prime}(\lambda, \alpha+\Delta \alpha, \delta)$ and $T_{c a l, i}^{\prime \prime \prime}(\lambda, \alpha, \delta+\Delta \delta) \quad(i=1 \sim \mathrm{N})$.

(4) Find $x_{\lambda}, x_{\alpha}$ and $x_{\delta}$ that satisfy the following equation:

$$
\begin{aligned}
S_{\text {linear }}=\sum_{i=1}^{N}\left[\left(T_{\text {exp }, i}-T_{c a l, i}\right)-x_{\lambda}\left(T_{c a l, i}^{\prime}-T_{c a l, i}\right)-\right. \\
\left.x_{\alpha}\left(T_{c a l, i}^{\prime \prime}-T_{c a l, i}\right)-x_{\delta}\left(T_{c a l, i}^{\prime \prime \prime}-T_{c a l, i}\right)\right]^{2}=\min
\end{aligned}
$$

(5) Set new values for $\lambda, \alpha$ and $\delta$ such that

$$
\lambda^{\text {new }}=\lambda+x_{\lambda} \Delta \lambda, \quad \alpha^{\text {new }}=\alpha+x_{\alpha} \Delta \alpha, \quad \delta^{\text {new }}=\delta+x_{\delta} \Delta \delta
$$

(6) Repeat steps (2)-(5).

The values of $\Delta \lambda, \Delta \alpha$ and $\Delta \delta$ were taken to be $1 \%$ of the current estimates $\lambda, \alpha$ and $\delta$. The step (4) was carried out with the linear least-square method using Gram-Schmidt ortho-normalization and Q-R factorization.

The first guess was chosen from a prepared data set of calculated temperature rise. The average temperature rise of the sensor has been calculated as a function of time for various combinations of $\lambda$ and $\alpha$, which are given in increments of $20 \%$, with a fixed thickness of the gel layer that equals to the cavity depth, $50 \mu \mathrm{m}$. The values of $\lambda$ and $\alpha$ that yield minimum 
difference in the temperature rise between calculation and experiment were taken for the first guess.

The standard deviation of the difference between the experimental data and calculated temperatures, i.e.

$$
S D_{\Delta T}=\sqrt{\frac{S}{N}}=\sqrt{\frac{1}{N} \sum_{i=1}^{N}\left(T_{\text {exp }, i}-T_{c a l, i}(\lambda, \alpha, \delta)\right)^{2}}
$$

was evaluated after step (4) in each iteration, and used as an index of convergence.

\subsection{Specifying time at the start of heating}

The measured data included some anomalous voltages at the start of heating probably caused by switching the range of applied current and 1-ms difference in timing between the measurement of voltage and current. This resulted in missing of one or two data, and made it difficult to specify the time at the start of heating. We therefore assumed the 'time zero', i.e. the time at the start of heating, at one interval of measurement, $0.18 \mathrm{~s}$, ahead of the point that exhibited first temperature rise. However, it didn't work for obtaining final results depending on repeated experiments. This is because the difference in the temperature between theory and experiment near the start of heating does not change significantly with changing $\lambda$ and $\alpha$. This implies that, to be more precise, the time zero should be taken into account as a variable in addition to $\lambda, \alpha$ and $\delta$. However since this is unrealistic from a technical point of view at present as a preliminary study, we determined the time zero with more straightforward way. The difference in time between the experimental data and the temperature rise calculated with first assumption of $\lambda$ and $\alpha$ was estimated for the first several points (Fig. 6). Then the time zero was shifted by $\Delta t$, which was taken as an average of first three, $\Delta t_{1}, \Delta t_{2}$ and $\Delta t_{3}$ in Fig. 6 , in the present study. 


\section{RESULTS AND DISCUSSIONS}

The process for determining the thermal conductivity and the thermal diffusivity is demonstrated in Figs. 7 and 8 and Table 1 for the measurement of acrylic resin. In this case, $\sim 5$-K rise in the temperature of the sensor within $5 \mathrm{~s}$ was obtained by heating at $\sim 20 \mathrm{~mW}$. The temperature change chosen from the prepared data set as an initial assumption was that calculated with $\lambda_{1}=0.244 \mathrm{~W} / \mathrm{mK}, \alpha_{1}=1.50 \times 10^{-7} \mathrm{~m}^{2} / \mathrm{s}$, and $\delta_{1}=50 \mu \mathrm{m}$. The calculated temperature rise was lower than the measured temperature by $\sim 0.08 \mathrm{~K}$ at $1 \mathrm{~s}$ and the difference increased gradually with time (Fig. 8). The thickness of the gel, $\delta$, was fixed at 50 $\mu \mathrm{m}$ for the following iteration steps as long as the difference between the calculated and measured temperature, $S D_{\Delta T}$, exceeded $0.03 \mathrm{~K}$. In the case shown in Figs. 7 and $8, \delta$ was incorporated as a variable at the third step after $S D_{\Delta T}$ reduced to $0.011 \mathrm{~K}$ at the second iteration (Table 1). While the difference between the measured and calculated temperatures increased once up to $0.149 \mathrm{~K}$ in $S D_{\Delta T}$ by incorporation of $\delta$, it decreased again with further iteration (Fig. 8 and Table 1). Iteration was stopped after 6th trial because of no reduction in $S D_{\Delta T}$ from the 5th trial. Although the calculated temperature rise agreed well with the measured temperature within the difference only $0.003 \mathrm{~K}$ in $S D_{\Delta T}$, the differences of finally determined values from the literature were $-3.0 \%$ for $\lambda,-15.4 \%$ for $\alpha$, and $-39.1 \%$ for $\delta$. The errors in $\lambda, \alpha$ and $\delta$ were different reflecting the difference in their effect on the temperature rise. However, the error does not necessarily result from the error in the temperature measurement or the numerical analysis. It is attributed in part to the difference between the real system and the model; the gel layer may not be completely uniform in thickness, or the heat conduction to the air from the backside surface may not be negligible. The error in determining the time at the start of heating could be also a cause of the error, which will be discussed later. 
Figures 9-11 show the measured temperature and the temperature rise obtained during the iteration process for other materials; agar gel, machinable ceramic, and SUS304. Because of the wide range of thermal transport properties, the heating power that gave appropriate temperature rise was considerably different each other depending on the samples. In addition, the increasing manner was also different particularly for SUS304; the temperature rise for SUS304 was much faster than the other after the start of heating but slowed down immediately (Fig. 11). Even though the experimental data showed variations in the transient temperature rise, the calculated temperature rises at the final iteration step agreed well with the experimental data in all cases. However, this does not imply that the finally determined transport properties agreed well with literature values.

The summary of finally obtained results for all tested materials is shown in Fig.12 and Table 2 with literature values. Five individual measurements were carried out for each material. Even though only one sample was used for a given material, the results depended on each measurement because of the difference in preparation and setting. This indicates a need for replication to obtain thermal transport properties with the present method. The final results were obtained after three to nine iterations. The thermal conductivity determined from the average of five replicates was different from the literature value by $-7.3 \%$ for acrylic resin, $6.2 \%$ for agar gel, $10.8 \%$ for machinable ceramic, and $-5.2 \%$ for SUS304. This indicates that the measurement error could be less than $10 \%$ irrespective of the material. However, significantly larger error was found in thermal diffusivity for acrylic resin and SUS304; the difference from the literature value was $-23.9 \%$ for acrylic resin and $-35.9 \%$ for SUS304, while it was only $-0.9 \%$ for agar gel and $-1.5 \%$ for machinable ceramic. This resulted from the fact that the temperature rise is less sensitive to the thermal diffusivity than the thermal conductivity, which has been demonstrated in the discussion with theoretical analysis [23]. 
We have used a method based on the Gauss-Newton algorithm to determine $\lambda, \alpha$ and $\delta$ iteratively. The method potentially has a problem of dependency on the initial value, which may lead to a completely different combination of $\lambda$ and $\alpha$ as a final solution. However, it was not the case in the present study irrespective of the wide range of $\lambda$ and $\alpha$ of tested materials by two orders of magnitude as shown in Fig. 12. This is probably because the initial values were determined from the prepared data set. The result therefore suggests that we are able to avoid serious errors by preparing a number of data sets with various combinations of $\lambda$ and $\alpha$ as a candidate of initial guess.

Considerably small values of $S D_{\Delta T}$, smaller than $0.02 \mathrm{~K}$ in most cases and $0.067 \mathrm{~K}$ even at the largest case, indicate that the temperature rise obtained with finally determined $\lambda$ and $\alpha$ agreed well with the measurement as shown in Figs. 7, 9, 10, and 11. Even so, the values of $\lambda$ and $\alpha$ did not always agree well with literature values. In addition, smaller $S D_{\Delta T}$ did not imply better result. This is probably because that modifying $\lambda$ and $\alpha$ does not significantly influence the difference between measured and calculated temperatures near the start of heating, and the difference here could be the major part of $S D_{\Delta T}$ at the final iteration step. This could be another reason for the scattering of $\lambda$ and $\alpha$ that depended on replicates, because the timing of the measurement relative to the actual start of heating was different in each experimental run. This suggests the importance of estimating the time zero to improve our method.

The measurement error was much larger than those estimated in our previous examination with virtual experimental data [23]. One of the reasons is that the measured temperature rises do have some errors in temperatures, whereas the virtual experimental data have taken into account only scattering. A deviation from the assumed situation in the model, uniform thickness of the gel layer for an example, could also happen in the real system. The other 
major difference is that the exact time for the start of heating has been given in our previous study. We therefore might be able to significantly reduce the measurement error by introducing a better method for specifying the time at the start of heating.

The thickness of the gel layer $\delta$ varied much more than $\lambda$ and $\alpha$, some of them were larger than the twice of the thickness of the spacer, and the others were smaller than the spacer thickness, which could not be the case theoretically. The scattering was the largest in the measurement of agar gel. This is reasonable because the gel has thermal transport properties similar to the agar gel; if thermal transport properties of the gel and the sample are identical, it is theoretically impossible to determine the thickness of the gel. The difficulty of determining $\delta$ is due to the fact that the temperature rise was not affected significantly by $\delta$, which has been also demonstrated in our previous study [23]. However, since the value of $\delta$ is no concern of the measurement, low sensitivity of the temperature rise to $\delta$ is preferable to the present method.

Even though the proposed method may need improvement, the smallest error was found in the measurement of agar gel. This suggests that our method has an advantage over the other methods in measuring thermal transport properties of soft materials.

\section{CONCLUSIONS}

The final goal of our research is to develop a method for measuring thermal transport properties using a novel stamp sensor. We have demonstrated the feasibility of the proposed method by preliminary experiments using a prototype sensor with four kinds of materials, i.e. acrylic resin, agar gel, machinable ceramic and SUS304, which have a wide range of thermal transport properties. The conclusions are as follows:

1. The thermal conductivity, the thermal diffusivity and the thickness of gel layer were determined successfully by the Gauss-Newton algorithm within three to nine iterations. 
The calculated temperature agreed well with the measured temperature rise of the sensor.

2. The thermal conductivity in the range from 0.21 to $16 \mathrm{~W} /(\mathrm{m} \cdot \mathrm{K})$ could be measured within $10 \%$ error, while the error in the thermal diffusivity ranged between $1.2 \times 10^{-7}$ and $3.6 \times 10^{-6} \mathrm{~m}^{2} / \mathrm{s}$ would be much larger, up to $35 \%$ for SUS304. The accuracy of the measurement is potentially improved by specifying more closely the time at the start of heating.

2. The method has an advantage in measuring soft materials over other conventional methods.

\section{ACKNOWLEDGEMENT}

This study was supported in part by Mayekawa Houonkai Foundation provided to HT in 2010, and Japan Science and Technology Agency through the program A-STEP (FS stage, 2011) to TF. Scholarship provided by Indonesia Government for Doctoral Degree at Kyushu University, Japan is greatly acknowledged by $\mathrm{SH}$, an academic staff of Sebelas Maret University, Indonesia.

\section{REFERENCES}

[1] W. Parker, R. Jenkins, C. Butler, and G. Abbott, Flash Method of Determining Thermal Diffusivity, Heat Capacity, and Thermal Conductivity, J. Appl. Phys. 32 (9) (1961) $1679-1684$.

[2] Akoshima, M., Hata, K., Futaba, D.N., Mizuno, K., Baba, T., and Yumura, M., Thermal Diffusivity of Single-Walled Carbon Nanotube Forest Measured by Laser Flash Method, Japanese Journal of Applied Physics 48 (2009) 05EC07.

[3] Baba, T., and Ono, A., Improvement of the Laser Flash Method to Reduce Uncertainty in Thermal Diffusivity Measurements, Measurement Science and Technology 12 (2001) 
2046-2057.

[4] Hemberger, F., Ebert, H.P., and Fricke, J., Determination of the Local Thermal Diffusivity on Inhomogenous Samples by a Modified Laser-Flash Method, International Journal of Thermophysics 28 (5) (2007) 1509-1521.

[5] He, Y., Rapid Thermal Conductivity Measurements with a Hot Disk Sensor, Part 1. Theoretical Considerations, Thermochimica Acta 436 (2005) 122-129.

[6] Gustafsson, S.E., Karawacki, E., and Khan, M. N., Transient Hot-strip Method for Simultaneously Measuring Thermal Conductivity and Thermal Diffusivity of Solids and Fluids, J. Phys. D: Appl. Phys. 12 (1979) 1411-1421.

[7] Gustafsson, S.E., Karawacki, E., and Chohan M.A., Thermal Transport Studies of Electrically Conducting Materials Using The Transient Hot-strip Technique, J. Phys. D: Appl. Phys. 19 (1986) 727-735.

[8] Gustafsson, S.E., Transient Plane Source Techniques for Thermal Conductivity and Thermal Diffusivity Measurements of Solids Materials, Rev. Sci. Instrum. 62 (3) (1991) 797-804.

[9] Karawacki, E., and Sulaiman, B.M., Dynamic Plane Source Technique for Simultaneous Determination of Specific Heat, Thermal Conductivity and Thermal Diffusivity of Metallic Samples, Meas. Sci. Technol. 2 (1991) 744-750.

[10] Gustavsson, M., Karawacki, E., and Gustafsson, S.E., Thermal Conductivity, Thermal Diffusivity, and Specific Heat of Thin Samples from Transient Measurements with Hot Disk Sensors, Rev. Sci. Instrum. 65 (12) (1994) 3856-3859.

[11] Log, T., and Gustafsson, S.E., Transient Plane Source (TPS) Technique for Measuring Thermal Transport Properties of Building Materials, Fire and Materials 19 (1995) 43-49.

[12] Bohac, V., Gustavsson, M.K., Kubicar, L., and Gustafsson, S.E., Parameter Estimations for Measurements of Thermal Transport Properties with The Hot Disk Thermal 
Constants Analyzer, Rev. Sci. Instrum. 71 (6) (2000) 2452-2455.

[13] Solorzano, E., Reglero, J.A., Rodriguez-Perez, M.A., Lehmhus, D., Wichman, M., and de Saja, J.A., An Experimental Study on the Thermal Conductivity of Aluminum Foams by Using the Transient Plane Source Method, International Journal of Heat and Mass Transfer 51 (2008) 6259-6267.

[14] Al-Ajlan, S.A., Measurements of Thermal Properties of Insulation Materials by Using Transient Plane Source Technique, Applied Thermal Engineering 26 (2006) 2184-2191.

[15] Anis-ur-Rehman, M., and Maqsood, A., Measurement of Thermal Transport Properties with an Improved Transient Plane Source Technique, International Journal of Thermophysics 24 (3) (2003) 867-883.

[16] Almanza, O., Rodriguez-Perez, M.A., and De Saja, J.A., Applicability of the Transient Plane Source Method To Measure the Thermal Conductivity of Low-Density Polyethylene Foams, Journal of Polymer Science Part B: Polymer Physics 42 (2004) 1226-1234.

[17] Kerr, L.L., Pan, Y., Dinwiddie, R.B., Wang, H., and Peterson, R.C., Thermal Conductivity of Coated Paper, Int. J Thermophysics 30 (2009) 572-579.

[18] Huang, L,. and Liu, L., Simultaneous Determination of Thermal Conductivity and Thermal Diffusivity of Food and Agricultural Materials Using a Transient Plane-Source Method, Journal of Food Engineering 95 (2009) 179-185.

[19] Lisker, I.S., Solovyev, S.V., Axcell, B.P., Varlow, B.R., and Donnelly, K., A Transient Technique for Measuring the Thermal Conductivity of Non-Metals, Experimental Thermal and Fluid Science 25 (2001) 377-382.

[20] Takahashi, I., and Emori, M., Measuring Method of Three Thermophysical Parameters of Solids by Thermal Probe with Instantaneous Point Contact (Measuring Principle)(in Japanese), Netsu Bussei 13 (4) (1999) 246-251. 
[21] Takahashi, I., and Emori, M., Measuring Method of Three Thermophysical Parameters of Solids by Thermal Probe with Instantaneous Point Contact (Discussion about Applicability and Measuring Conditions)(in Japanese), Netsu Bussei 13 (4) (1999) $252-257$.

[22] Okabe, T., Mashimo, H., Okajima, J., Komiya, A., Takahashi, I., and Maruyama, S., A Measurement Method of Thermal Conductivity of Soft Materials and Liquids by Utilizing Point-Contact Method (in Japanese), Netsu Bussei 26 (3) (2012) 136-141.

[23] Hadi, S., Nishitani, M., Wijayanta, A.T., Kurata, K., and Takamatsu, H., Measurement of Thermal Conductivity and Thermal Diffusivity of Solid Materials Using a Novel Stamp Sensor: A Feasibility Study with Numerical Analysis, Journal of Thermal Science and Technology JSME 7 (4) (2012) 536-548.

[24] Woodfield, P.L., Fukai, J., Fujii, M., Takata, and Y., Shinzato, K., Determining Thermal Conductivity and Thermal Diffusivity of Low-Density Gases Using the Transient Short-Hot-Wire Method, Int. J. Thermophysic 29 (2008) 1299-1320.

[25] JSME Data Book : Heat Transfer $5^{\text {th }}$ edition (in Japanese), 2009, pp. 285, 288.

[26] Tanaka, T., Measurement of Thermal Conductivity and Thermal Diffusivity of Agar Gel Using Short-Hot-Wire Method (in Japanese), Master Thesis, Kyushu University, 2008.

\section{NOMENCLATURE}

i : Number of iteration

$Q \quad$ : Heating power $(\mathrm{W})$ 
$S D_{\Delta \mathrm{T}}$ : Standard deviation of difference between the calculated and measured temperatures $(\mathrm{K})$

$T \quad$ : Temperature (K)

$t \quad$ : Time (s)

$t_{0} \quad$ : Time at the start of heating (s)

$\alpha \quad$ : Thermal diffusivity $\left(\mathrm{m}^{2} / \mathrm{s}\right)$

$\Delta t \quad:$ Difference in time (s)

$\delta \quad:$ Thickness of gel layer (m)

$\lambda \quad:$ Thermal conductivity $(\mathrm{W} / \mathrm{m} \mathrm{K})$

\section{SUBSCRIPTS}

cal : calculated or theoretically obtained value

$\exp :$ experiment

ref : reference 


\section{Figure legends}

Table 1 Change of $\lambda, \alpha$, and $\delta$ during iteration process

Table 2 Measured values of thermal conductivity and thermal diffusivity

Fig. 1 Schematic of stamp sensor; (a) Cross-section, (b) External appearance

Fig. 2 Pattern of the sensor deposited on the glass substrate

Fig. 3 Prototype sensor; (a) External appearance, (b) Cross-section

Fig. 4 Schematic of measurement system

Fig. 5 Physical model and boundary conditions

Fig. 6 Difference in temperature rise near the start of heating between measurement and calculation with initially assumed time of heating.

Fig. 7 Measured temperature rise for acrylic resin and calculated values at the iteration process

Fig. 8 Change of the difference between measured and calculated temperature rise during iteration process

Fig. 9 Measured and calculated temperature rise for agar gel

Fig. 10 Measured and calculated temperature rise for machinable ceramics

Fig. 11 Measured and calculated temperature rise for SUS304

Fig. 12 Comparison of measured thermal conductivity and thermal diffusivity with literature values 
Table 1 Change of $\lambda, \alpha$, and $\delta$ during iteration steps

\begin{tabular}{|c|c|c|c|c|c|c|c|}
\hline$i$ & $\begin{array}{c}\lambda \\
(W / m K)\end{array}$ & $\begin{array}{c}\alpha \\
\left(10^{-7} \mathrm{~m}^{2} / \mathrm{s}\right)\end{array}$ & $\begin{array}{c}\delta \\
(\mu \mathrm{m})\end{array}$ & $\begin{array}{c}\frac{\lambda-\lambda_{\text {ref }}}{\lambda_{\text {ref }}} \\
(\%)\end{array}$ & $\begin{array}{c}\frac{\alpha-\alpha_{r e f}}{\alpha_{r e f}} \\
(\%)\end{array}$ & $\begin{array}{c}\frac{\boldsymbol{\delta}-\boldsymbol{\delta}_{r e f}}{\boldsymbol{\delta}_{r e f}} \\
(\%)\end{array}$ & $\begin{array}{r}S D_{\Delta \mathrm{T}} \\
(\mathrm{K})\end{array}$ \\
\hline $1 \mathrm{st}$ & 0.244 & 1.50 & 50 & 16.2 & 25.0 & -16.7 & 0.128 \\
\hline 2nd & 0.238 & 1.67 & 50 & 13.1 & 39.4 & -16.7 & 0.011 \\
\hline $3 \mathrm{rd}$ & 0.204 & 0.85 & 36.3 & -2.8 & -29.4 & -39.5 & 0.149 \\
\hline 4 th & 0.204 & 1.01 & 36.8 & -2.7 & -15.9 & -38.8 & 0.013 \\
\hline 5 th & 0.204 & 1.02 & 36.5 & -3.0 & -15.4 & -39.1 & 0.003 \\
\hline 6 th & 0.204 & 1.02 & 36.5 & -3.0 & -15.4 & -39.1 & 0.003 \\
\hline
\end{tabular}


Table 2 Measured values of thermal conductivity and thermal diffusivity

\begin{tabular}{|c|c|c|c|c|c|c|c|c|}
\hline Material & $\underset{(\mathrm{mW})}{Q}$ & $i$ & $\begin{array}{c}\lambda \\
(W / m K)\end{array}$ & $\begin{array}{c}\alpha \\
\left(\mathbf{x ~ 1 0} 0^{-7} \mathrm{~m}^{2} / \mathrm{s}\right)\end{array}$ & $\begin{array}{c}\delta \\
(\mu \mathrm{m})\end{array}$ & $\begin{array}{c}\frac{\lambda-\lambda_{\text {ref }}}{\lambda_{\text {ref }}} \\
(\%) \\
\end{array}$ & $\begin{array}{c}\frac{\alpha-\alpha_{r e f}}{\alpha_{r e f}} \\
(\%)\end{array}$ & $\begin{array}{c}S D_{\Delta T} \\
\text { (K) }\end{array}$ \\
\hline \multirow{5}{*}{$\begin{array}{c}\text { Acrilic Resin } \\
\lambda_{\text {ref }}=0.210 \mathrm{~W} / \mathrm{mK} \\
\alpha_{\text {ref }}=1.20 \times 10^{-7} \mathrm{~m}^{2} / \mathrm{s} \\
{[25]}\end{array}$} & 19.9 & 6 & 0.204 & 1.02 & 37 & -3.0 & -15.4 & 0.003 \\
\hline & 19.7 & 5 & 0.194 & 0.93 & 32 & -7.4 & -22.3 & 0.004 \\
\hline & 19.7 & 8 & 0.195 & 0.95 & 33 & -7.1 & -20.9 & 0.004 \\
\hline & 21.3 & 4 & 0.188 & 0.81 & 49 & -10.5 & -32.5 & 0.004 \\
\hline & 21.3 & 6 & 0.192 & 0.86 & 56 & -8.6 & -28.2 & 0.005 \\
\hline average & 20.4 & 6 & 0.195 & 0.9 & 41 & -7.3 & -23.9 & 0.004 \\
\hline \multirow{5}{*}{$\begin{array}{c}\text { Agar } \\
\lambda_{\text {ref }}= \\
0.58 \mathrm{~W} / \mathrm{mK} \\
\alpha_{\mathrm{ref}}=1.32 \times 10^{-7} \mathrm{~m}^{2} / \mathrm{s} \\
{[26]}\end{array}$} & 30.7 & 5 & 0.61 & 1.30 & 75 & 6.0 & -1.9 & 0.024 \\
\hline & 36.2 & 3 & 0.61 & 1.24 & 83 & 5.3 & -6.0 & 0.009 \\
\hline & 36.2 & 8 & 0.61 & 1.33 & 36 & 5.7 & 0.7 & 0.011 \\
\hline & 36.2 & 8 & 0.59 & 1.25 & 184 & 7.2 & 0.1 & 0.045 \\
\hline & 37.0 & 5 & 0.62 & 1.36 & 133 & 6.7 & 2.8 & 0.067 \\
\hline average & 35.3 & 6 & 0.61 & 1.30 & 102 & 6.2 & -0.9 & 0.031 \\
\hline \multirow{5}{*}{$\begin{array}{c}\text { Machinable } \\
\text { Ceramics } \\
\lambda_{\text {ref }}=1.60 \mathrm{~W} / \mathrm{mK} \\
\alpha_{\text {ref }}=7.84 \times 10^{-7} \mathrm{~m}^{2} / \mathrm{s} \\
{[25]}\end{array}$} & 50.8 & 5 & 1.86 & 7.38 & 100 & 16.0 & -5.9 & 0.002 \\
\hline & 50.8 & 3 & 1.81 & 7.72 & 82 & 13.3 & -1.5 & 0.009 \\
\hline & 50.8 & 4 & 1.76 & 7.74 & 69 & 10.0 & -1.3 & 0.001 \\
\hline & 50.8 & 6 & 1.80 & 7.60 & 110 & 12.7 & -3.0 & 0.001 \\
\hline & 50.9 & 4 & 1.63 & 8.18 & 75 & 2.1 & 4.4 & 0.002 \\
\hline average & 50.8 & 4 & 1.77 & 7.73 & 87 & 10.8 & -1.5 & 0.003 \\
\hline \multirow{5}{*}{$\begin{array}{c}\text { SUS304 } \\
\lambda_{\text {ref }}=16.3 \mathrm{~W} / \mathrm{mK} \\
\alpha_{\mathrm{ref}}=3.62 \times 10^{-6} \mathrm{~m}^{2} / \mathrm{s} \\
{[25]}\end{array}$} & 124.5 & 4 & 16.2 & 22.7 & 80 & -0.9 & -44.2 & 0.009 \\
\hline & 117.5 & 8 & 15.8 & 23.4 & 76 & -3.1 & -35.3 & 0.010 \\
\hline & 117.6 & 8 & 15.1 & 25.1 & 91 & -7.6 & -30.6 & 0.016 \\
\hline & 117.6 & 9 & 15.1 & 25.3 & 87 & -7.6 & -30.2 & 0.016 \\
\hline & 117.5 & 9 & 15.2 & 24.7 & 84 & -6.9 & -39.3 & 0.015 \\
\hline average & 119.0 & 8 & 15.5 & 24.3 & 84 & -5.2 & -35.9 & 0.013 \\
\hline
\end{tabular}




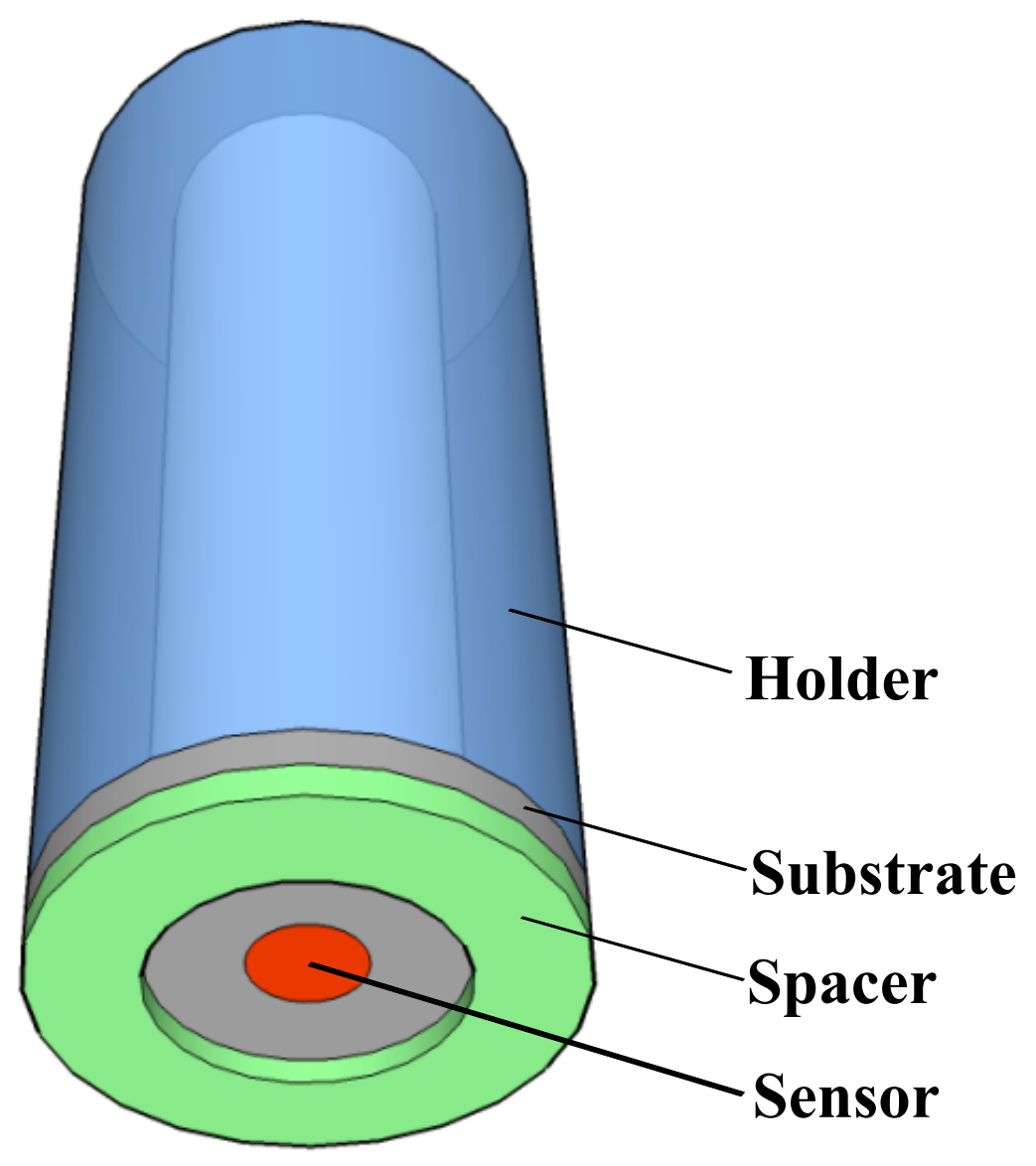

Fig. 1 Schematic of stamp sensor 

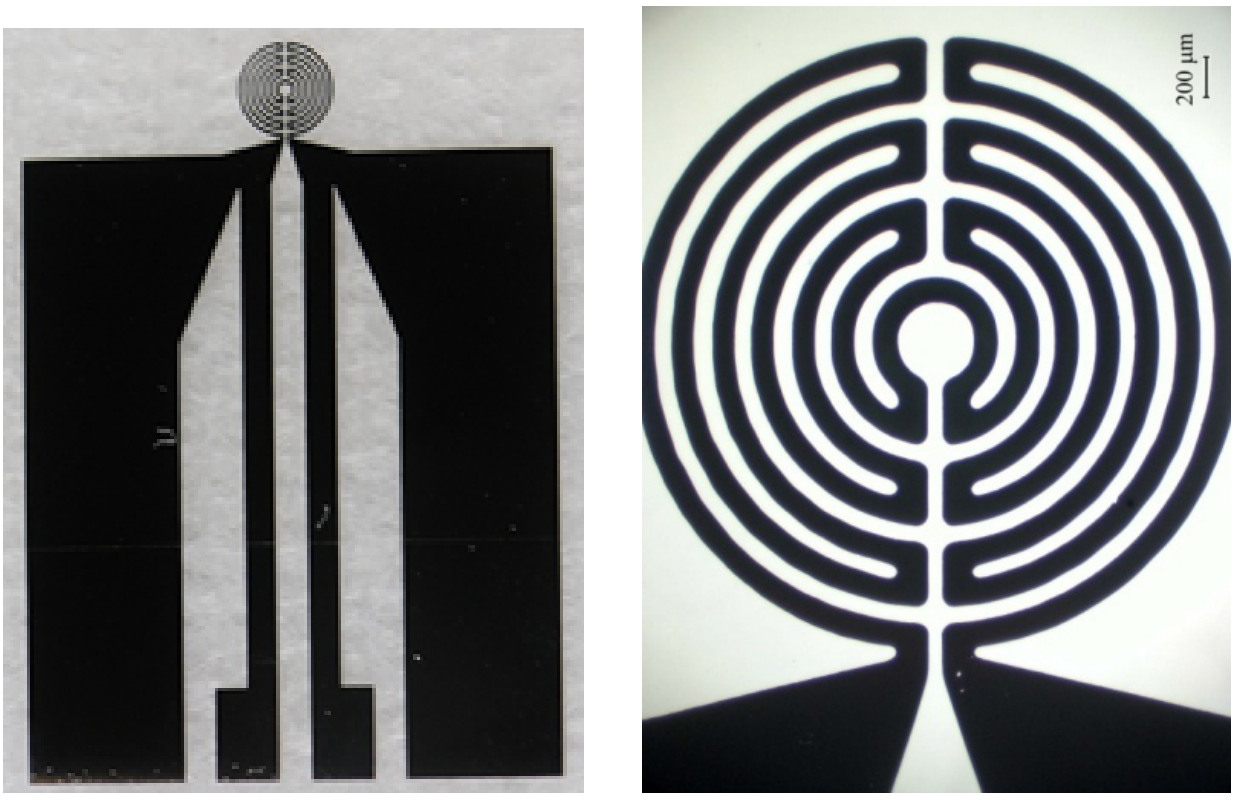

Fig. 2 Pattern of the sensor deposited on the glass substrate 


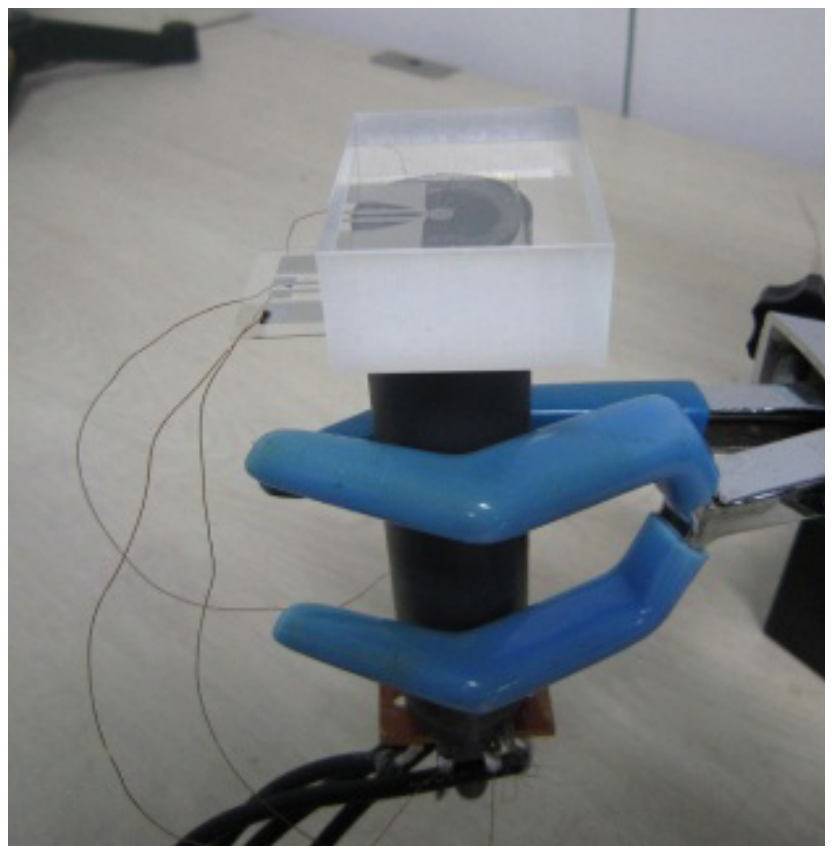

(a) External Appearance

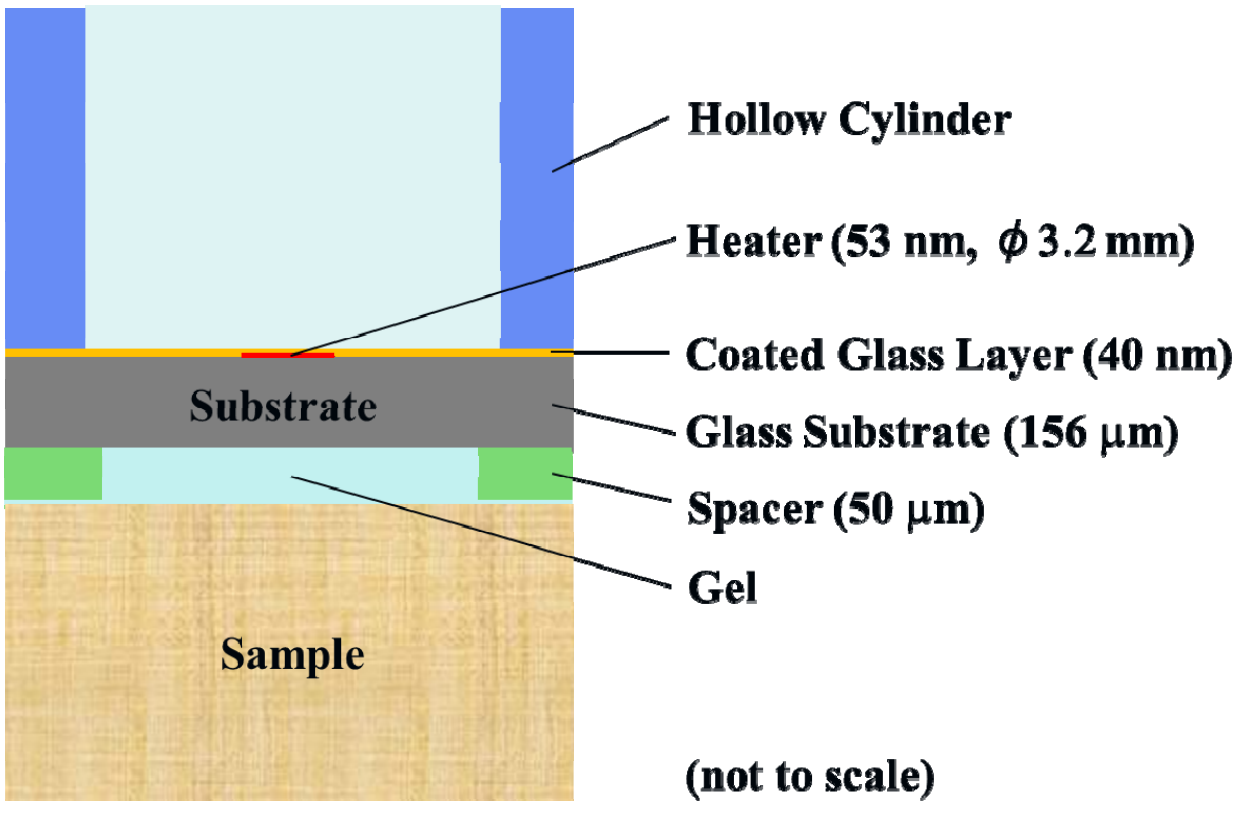

(b) Cross-section

Fig. 3 Prototype Sensor 


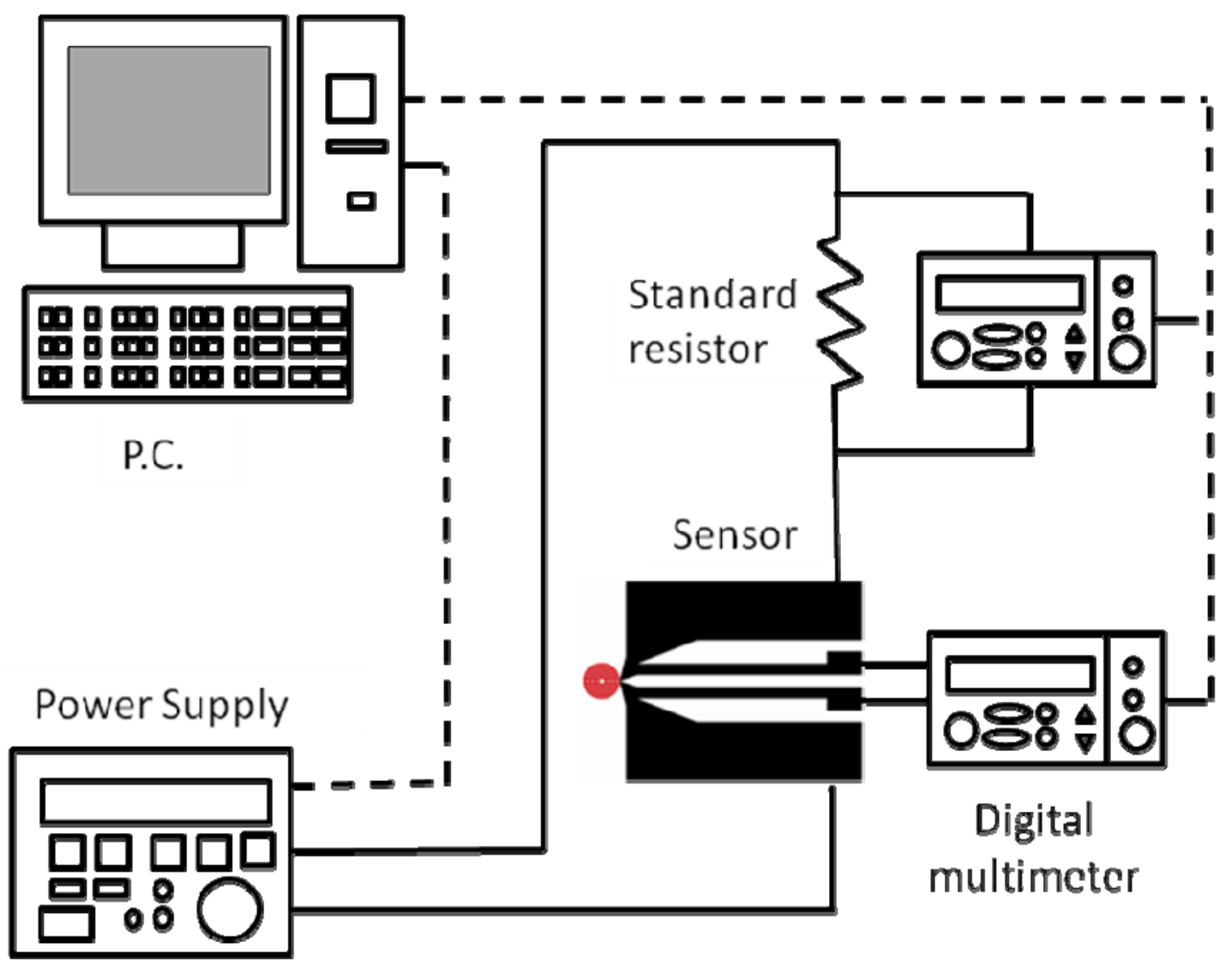

Fig. 4 Schematic of measurement system 


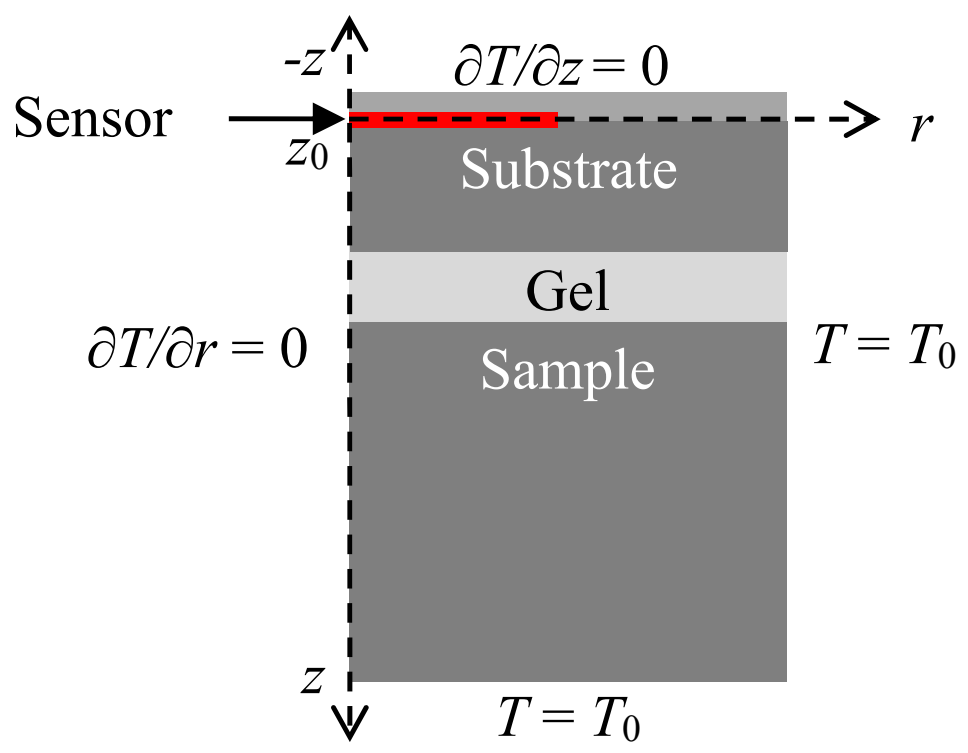

Fig. 5 Physical model and boundary conditions 


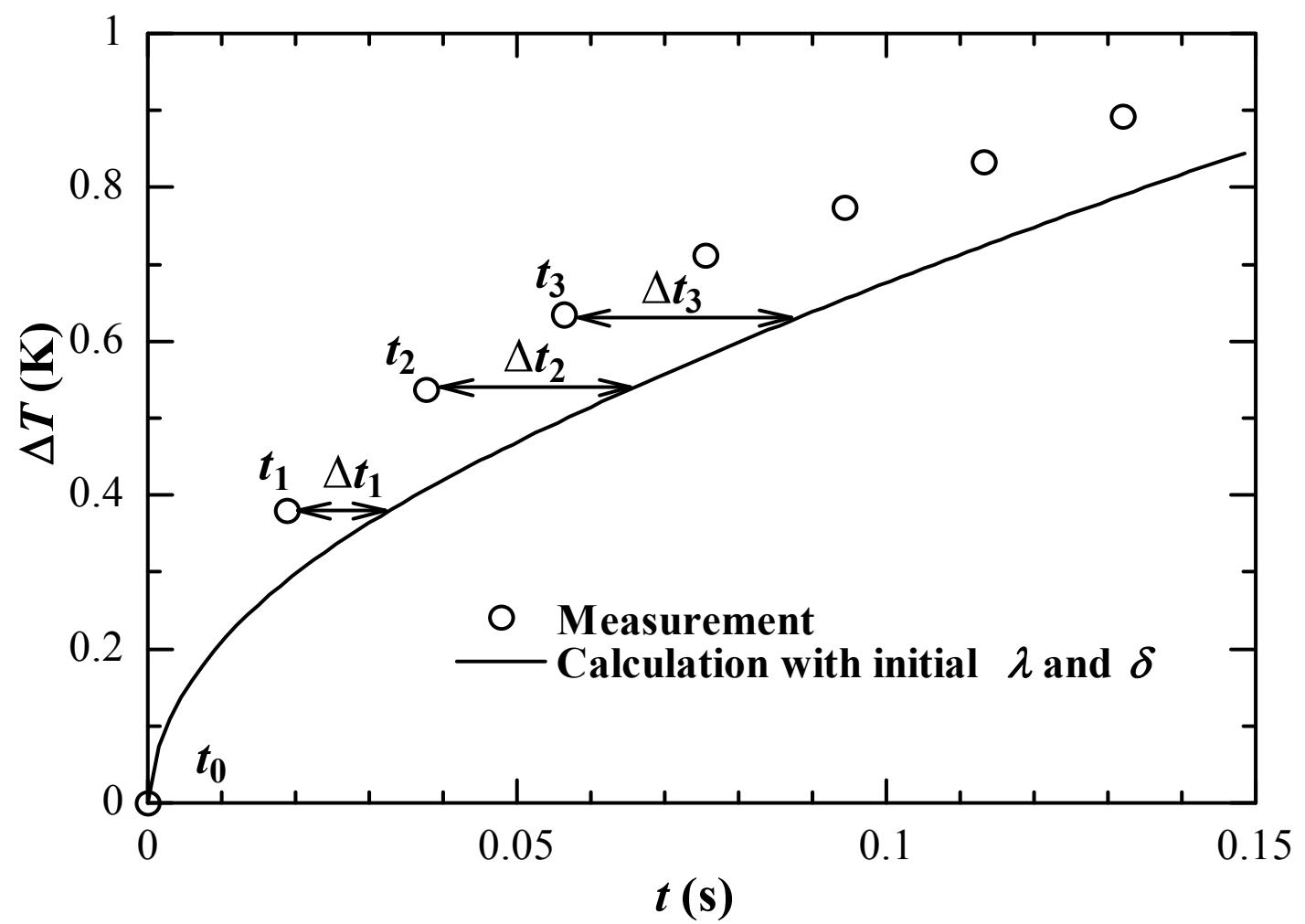

Fig. 6 Difference in temperature rise near the start of heating between measurement and calculation with initially assumed time heating 


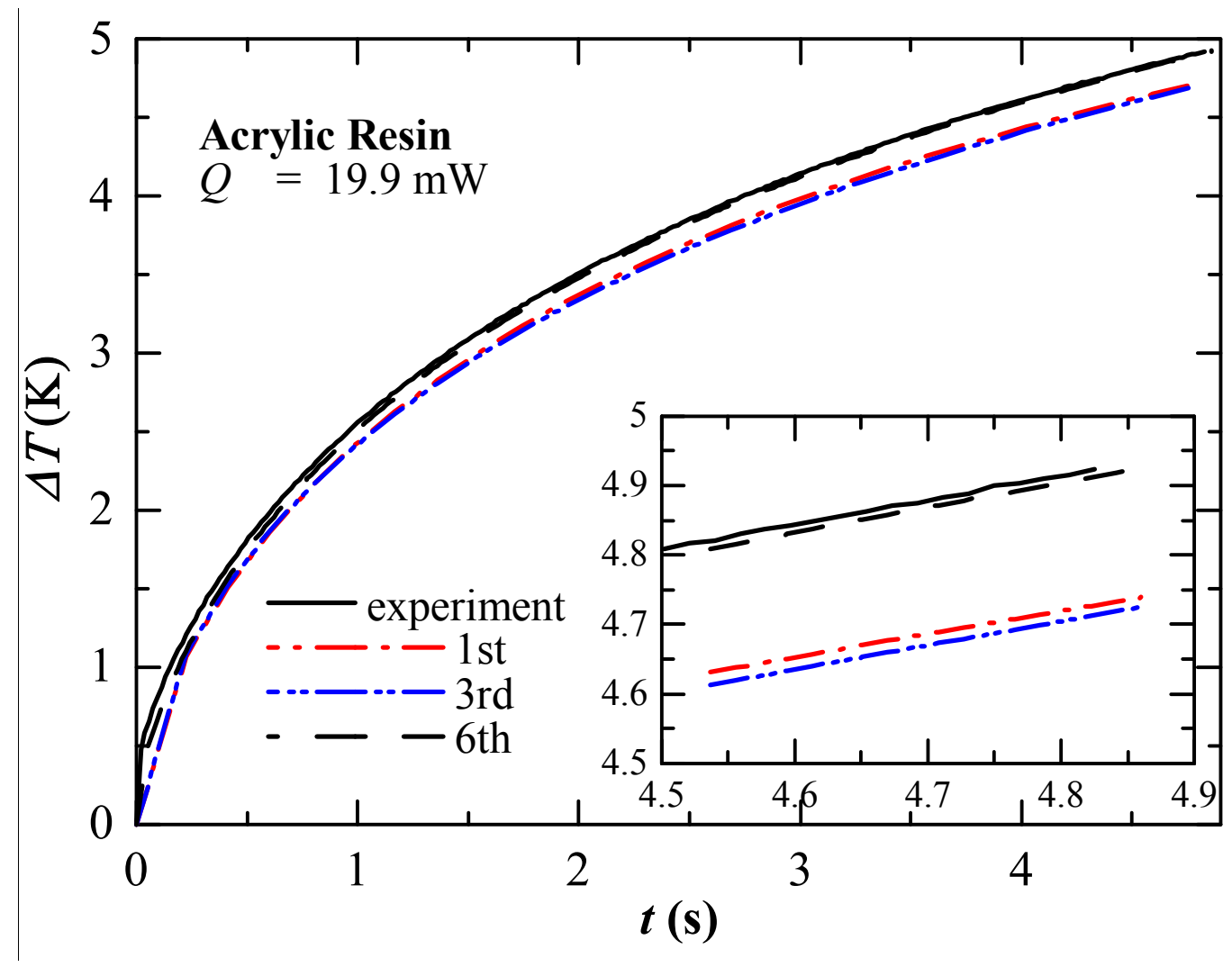

Fig. 7 Measured temperature rise for acrylic resin and calculated values at the iteration process 


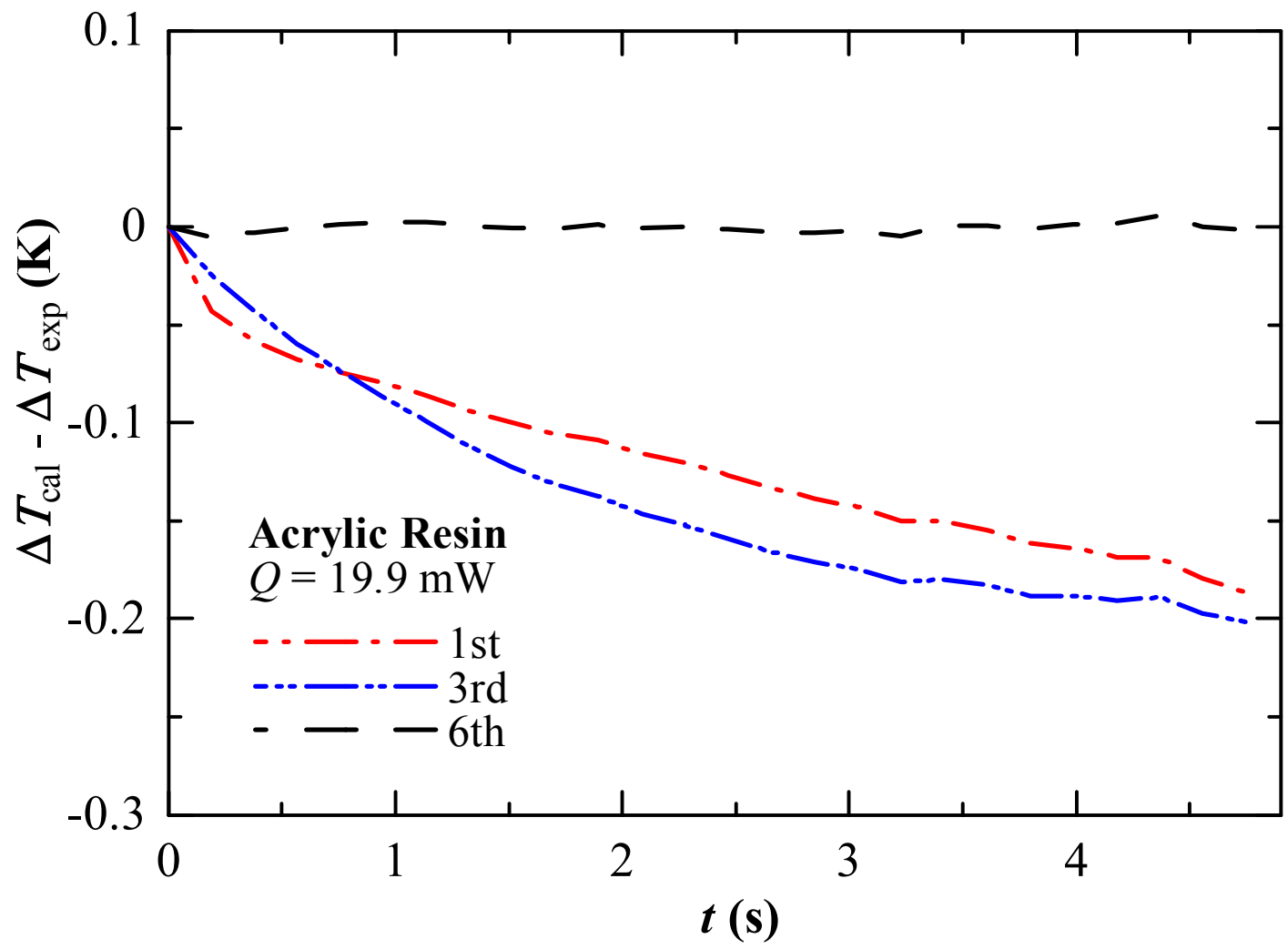

Fig. 8 Change of the difference between measured and calculated temperature rise during iteration process 


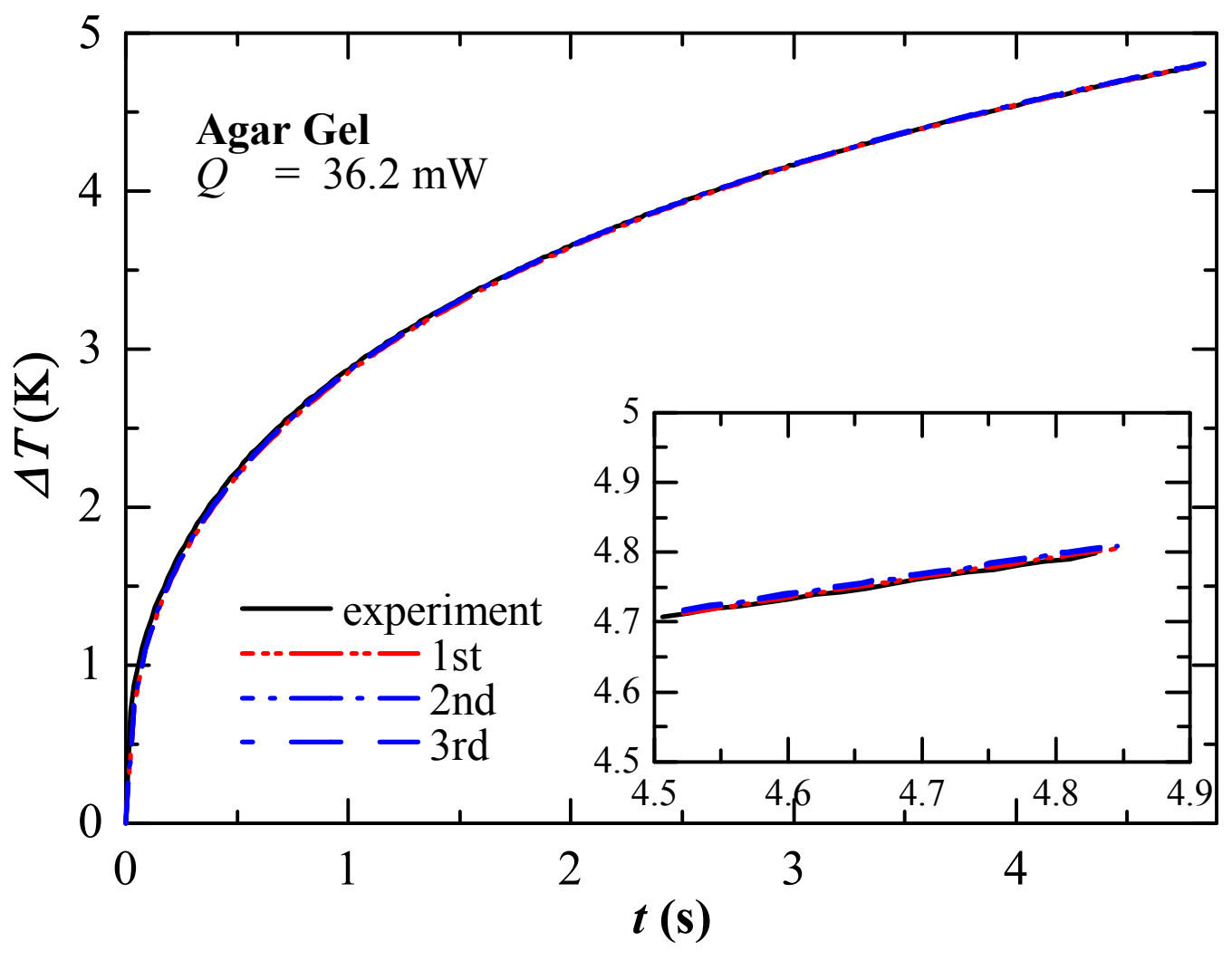

Fig. 9 Measured and calculated temperature rise for agar gel 


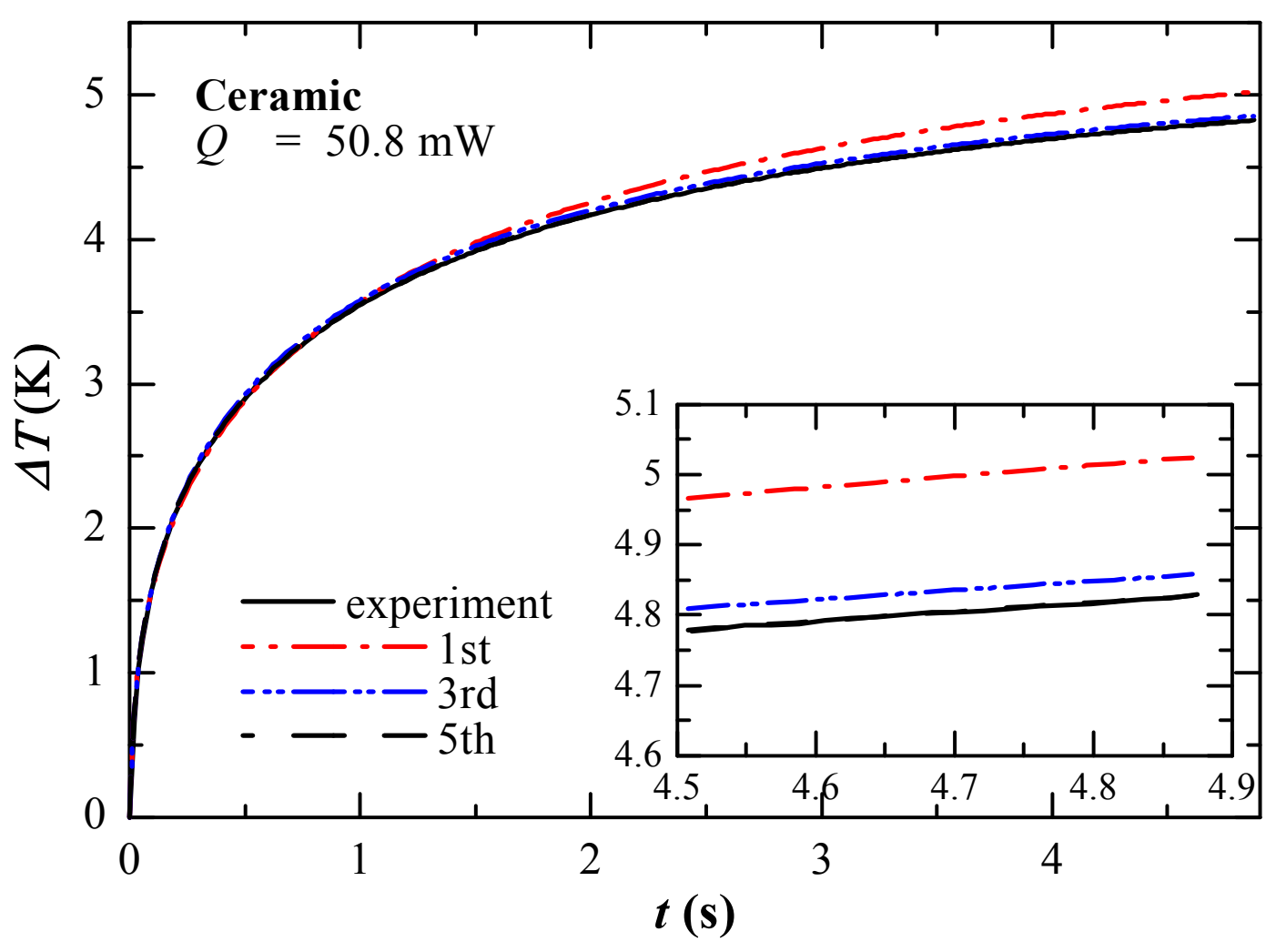

Fig. 10 Measured and calculated temperature rise for machinable ceramics 


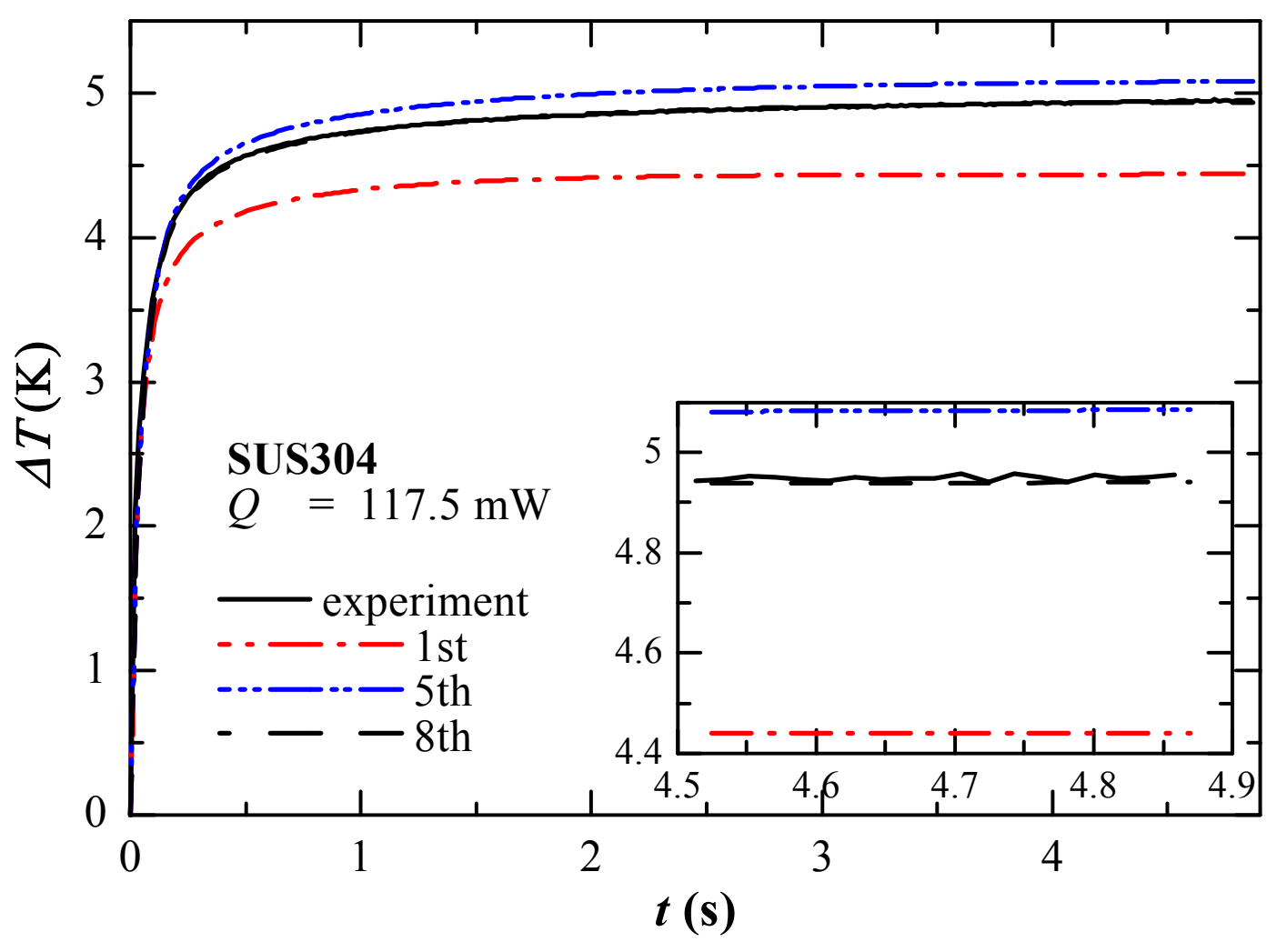

Fig. 11 Measured and calculated temperature rise for SUS304 


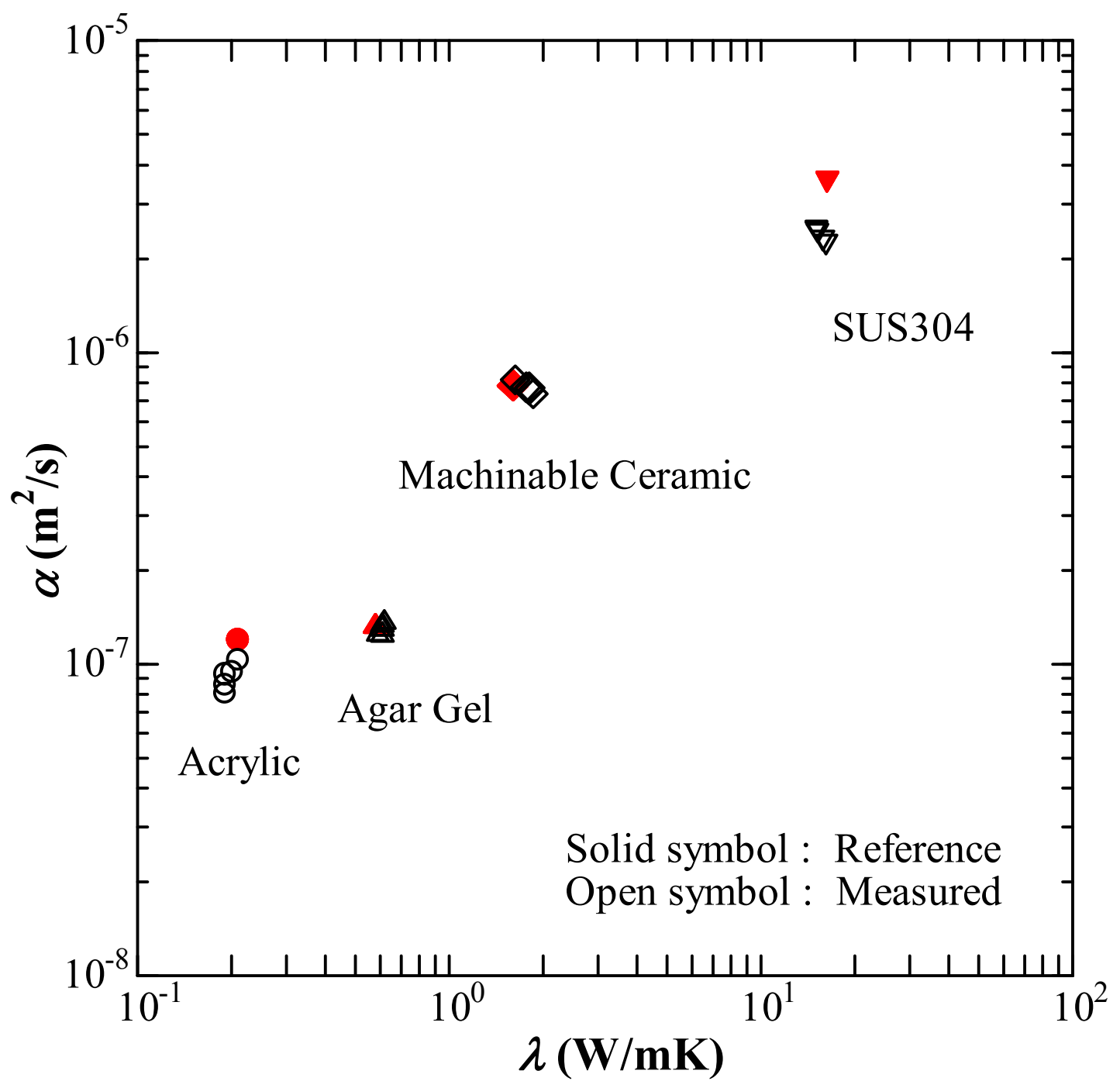

Fig. 12 Comparison of measured thermal conductivity and thermal diffusivity with literature values 